ARTICLE

Received 10 Jun 2013 | Accepted 3 Sep 2013 | Published 30 Sep $2013 \quad$ DOI: 10.1038/ncomms3538

\title{
Model studies of force-dependent kinetics of multi-barrier reactions
}

Yancong Tian ${ }^{1,2, \star}$, Timothy J. Kucharski ${ }^{2, \star}$, Qing-Zheng Yang $^{3} \&$ Roman Boulatov ${ }^{1,2}$

According to transition state theory, the rate of a reaction that traverses multiple energy barriers is determined by the least stable (rate-determining) transition state. The preceding ('inner') energy barriers are kinetically 'invisible' but mechanistically significant. Here we show experimentally and computationally that the reduction rate of organic disulphides by phosphines in water, which in the absence of force proceeds by an equilibrium formation of a thiophosphonium intermediate, measured as a function of force applied on the disulphide moiety yields a usefully accurate estimate of the height of the inner barrier. We apply varying stretching force to the disulphide by incorporating it into a series of increasingly strained macrocycles. This force accelerates the reduction, even though the strain-free rate-determining step is orthogonal to the pulling direction. The observed rate-force correlation is consistent with the simplest model of force-dependent kinetics of a multibarrier reaction.

\footnotetext{
${ }^{1}$ Department of Chemistry, University of Liverpool, Liverpool L69 7ZD, UK. ${ }^{2}$ Department of Chemistry, University of IIlinois, Urbana, Illinois 61801, USA

${ }^{3}$ Technical Institute of Physics and Chemistry, Chinese Academy of Sciences, Beijing 100190, China. * These authors contributed equally to this work.

Correspondence and requests for materials should be addressed to R.B. (email: r.boulatov@liv.ac.uk).
} 
E: xtensive empirical evidence indicates that kinetic stabilities of diverse reactive sites within a polymer are affected when the polymer is stretched by application of tensile force. Such force-dependent reactivity is thought to account for the behaviour of polymeric materials, melts and solutions under load, may be exploited to yield new stress-responsive, actuating and energy transducing materials $s^{1-10}$ and may even allow characterization of transition states. ${ }^{11}$ Considerable theoretical, ${ }^{3,12-19}$ computational ${ }^{13,20-26}$ and experimental ${ }^{27-34}$ effort has been devoted to refine and validate a model of forcedependent kinetics for elementary (single-barrier) reactions. Yet many reactions proceed through one or more intermediates, that is, multiple activation barriers separate the reactant(s) from the product(s). Examples include solvolysis of esters and amides (the $\mathrm{A}_{\mathrm{AC}} 2$ and $\mathrm{B}_{\mathrm{AC}} 2$ mechanisms), nucleophilic displacements (D and A mechanisms), additions, eliminations, cycloreversions, retroDiels-Alder and redox reactions. ${ }^{35,36}$

Little experimental or theoretical work has been reported on how stretching force affects the kinetics and mechanisms of multi-barrier reactions. Non-linear correlations between stretching force and the apparent activation energy of unfolding of several biopolymers was interpreted as indicating force-induced shift of the rate-determining step to an inner barrier (that is, a barrier that in the absence of force precedes the rate-determining step), but alternative explanations were proposed as well. ${ }^{37-44}$

A major theoretical challenge of extending the current models of force-dependent kinetics to multi-barrier reactions is the likelihood that stretching force affects individual steps of such reactions very differently because molecular dimensions change non-monotonically along the reaction paths. A stretched polymer in single-molecule force or elongational flow experiments can be thought of as a molecule coupled, at two of its atoms, to a constraining potential that exerts a stretching force on these two atoms. ${ }^{3,5,13,18,45,46} \mathrm{An}$ activation barrier for a reaction in such a polymer will depend on stretching force if the strain energy of the constraining potential is different at the top and the bottom of the barrier (that is, at the transition state and reactant or intermediate). To a good approximation, this strain energy change between two points on the energy surface (also known as work potential) is proportional to the difference of the separation of the two atoms at which the potential acts (so called pulling axis) at these points. For example, if the separation of the constrained atoms remains the same in a transition state and the corresponding reactant or the intermediate (that is, the pulling axis is orthogonal to the reaction coordinate), a stretching force will not affect the kinetics of that step. Even during multi-barrier molecular dissociations, certain internuclear distances that increase to infinity in the products may not change or even contract over some elementary steps. Figure 1 illustrates the diversity of patterns of changes in the terminal $\mathrm{C} \cdots \mathrm{C}$ distance along the steepest-descent paths of several double-barrier dissociation reactions of potential importance in the development of stress-responsive polymers.

Hydrolysis of esters and amides (red and black lines, Fig. 1) represents the simplest case of force-dependent kinetics of multibarrier dissociations: the first of the two barriers is both rate determining and orthogonal to the pulling axis (that is, not accompanied by changes in the terminal $\mathrm{C}^{\cdots} \mathrm{C}$ separation). Consequently, the dissociation rate is expected to be forceindependent, in agreement with reported computational and experimental data. ${ }^{47,48}$ In comparison, tensile force probably inhibits dissociation of methylethylidene cyclobutane (blue, Fig. 1).

A very interesting situation arises if the second barrier is rate determining in the absence of strain. Such reactions may manifest force-dependent kinetics even if this second step is orthogonal to the pulling axis and the first (or 'inner') barrier may become ratedetermining above a threshold force as illustrated in Fig. 2.

Here we report an atomistically tractable model reaction to test these predictions. We demonstrate that the shape of the experimental force-rate correlation is characteristic of a shift of the rate-determining step to the 'inner' barrier above a threshold force and the force-rate correlation above this force yields usefully accurate estimate of the strain-free rate constant for the formation of the intermediate. The latter is important because for most reactions that proceed by an equilibrium formation of a reaction intermediate, the parameters of this 'inner' barrier cannot be extracted from bulk kinetic measurements.

\section{Results}

Strategy. As highlighted recently, ${ }^{5}$ the relationship between the kinetic stability of a reactive moiety and the restoring force of its molecular coordinates is often independent of whether the moiety is part of a stretched polymer or a suitably designed strained macrocycle. This independence enables predictions of chemically driven micromechanical behaviour of polymers and experimental tests of models of such behaviour using simple non-polymeric substrates. $^{32}$ Here we used this approach to study forcedependent kinetics of reduction of aliphatic disulphides, RSSR, by phosphines, $\mathrm{PR}_{3}^{\prime}$, in aqueous medium. The strain-free mechanism is shown in Fig. $3 a{ }^{49-52}$ in neutral solution, the reduction of unstrained disulphides proceeds by rate-determining hydrolysis of a thiophosphonium intermediate. Because reversion

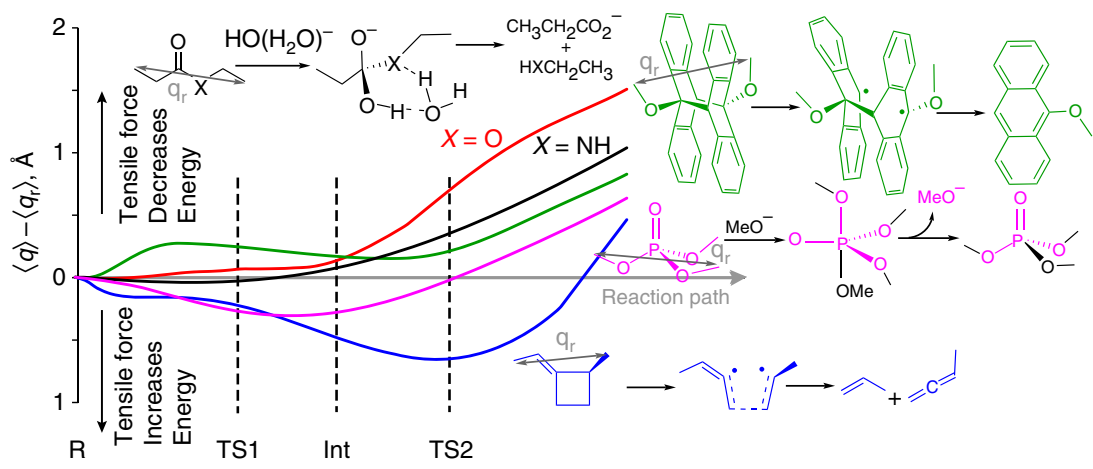

Figure 1 | Non-monotonous change of terminal distances along the reaction paths. The reaction schemes show the reactant, the intermediate and the product and the plotted distance (grey arrows) ${ }^{1,3,7,8}$. The computational details are described in the Methods; $\langle$ signifies Boltzmann averaging over multiple conformers comprising the stationary states; for degenerate exchange of $\mathrm{CH}_{3} \mathrm{O}^{-}$in $\mathrm{O}=\mathrm{P}\left(\mathrm{OCH}_{3}\right)_{3}$, the separation between the leaving group and each spectator $\mathrm{CH}_{3}$ group was included in calculating $\langle q\rangle$. 


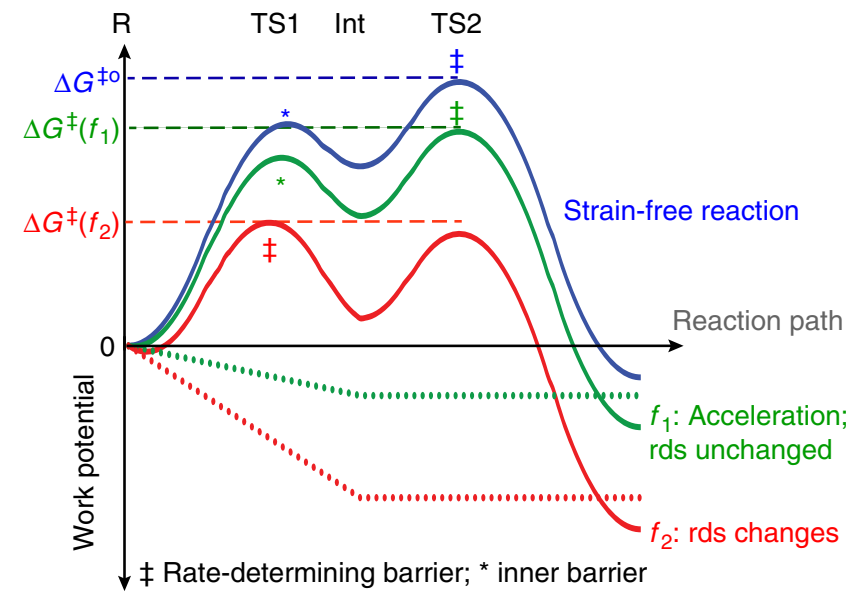

Figure 2 | The simplest model of a double-barrier reaction in the presence of force. The example has the second step orthogonal to the pulling axis. The inner barrier $\left(^{\star}\right)$ in the absence of force (blue curve) becomes rate determining $(\ddagger)$ when sufficient force is imposed across a suitable pair of the atoms of the reactant (red curve). Because the second step is orthogonal to the pulling axis (that is, the constrained distance does not change between the intermediate and second transition state), the ratedetermining step changes at applied force $\geq f_{2}$ because the force stabilizes the intermediate more than the first transition state. Consequently, the intermediate has to scale a larger barrier to revert to the reactants than to yield the product. Smaller force $f_{1}$ does not change the reaction mechanism but still accelerates the reaction by suppressing reversion of the intermediate to the reactant. The reaction energy profiles at $f_{1}$ and $f_{2}$ (green and red lines) are obtained by adding the change in the strain energy of the constraining potential ('work potential', dotted lines) to the reaction profile in the absence of force (blue line).

of this intermediate to the disulphide requires a thiolate nucleophile, which can be (partially) protonated by acid, reduction of disulphides by phosphines is one of the few reactions in which the rate constant for the formation of a highly unstable reaction intermediate can be measured. By comparing this rate constant derived from the $\mathrm{pH}$-dependence of the reduction rate of an unstrained disulphide with the one derived from the rate versus force correlations for a series of strained disulphides using the model in Fig. 2, the model can be tested experimentally.

We mimicked the strain that would be imposed on the disulphide moiety in a stretched polymer using a series of increasingly strained macrocyclic disulphides, E1-E6 (Fig. 3b). We previously demonstrated that when the strain of a reactive moiety in such macrocycles is quantified as a restoring force of a properly chosen local molecular coordinate, the rate-force correlations measured with a series of macrocycles and in polymers stretched in single-molecule force experiments are equivalent. ${ }^{32}$ Importantly, although the first step of reduction (formation of the thiophosphonium zwitterion) is coupled to changes in the molecular geometry of the stiff stilbene moiety (red, Fig. 3b), the second (hydrolysis) step is not. In other words, the pulling axis is orthogonal to the rate-determining elementary step in the absence of force. This orthogonality is maintained even if the reaction occurs in a polymer stretched externally. We resorted to studying a non-polymeric disulphide because mechanistic and computational studies required to test models of force-dependent reactivity cannot yet be performed on polymers.

Synthesis and kinetic measurements. We synthesized strain-free $Z$ disulphides (Fig. $3 \mathrm{~b}$ ), which served as reference for analysis of the reduction kinetics of the strained $E$ analogues (see below), following the scheme in Fig. 4.We measured the kinetics of reduction of these strain-free disulphides with large excess of triphenylphosphine $\left(\mathrm{PPh}_{3}\right)$ or methyldiphenylphosphine $\left(\mathrm{PPh}_{2} \mathrm{CH}_{3}\right)$ in $\mathrm{H}_{2} \mathrm{O}: \mathrm{CH}_{3} \mathrm{CN}(3: 1 \mathrm{~mol})$ at $60^{\circ} \mathrm{C}$ under rigorously anaerobic conditions. The compositions of all reaction mixtures were monitored for at least three half-lives by reverse-phase HPLC with aqueous $\mathrm{CH}_{3} \mathrm{CN}$ as eluent using an inert internal standard (see Supplementary Tables S3 and S4 for retention times and separation conditions). At $\mu \mathrm{M}$ concentrations of the cyclic disulphide, the reactions were first order in both phosphine and disulphide and yielded dithiol and phosphine oxides as the sole products, as confirmed independently by ${ }^{1} \mathrm{H}-\mathrm{NMR}$ and HRMS measurements. No reaction was observed in the absence of $\mathrm{H}_{2} \mathrm{O}$ and no intermediate could be detected either in aqueous or anhydrous $\mathrm{CH}_{3} \mathrm{CN}$, which is consistent with density functional theory (DFT) calculations of the free energy of formation of the zwitterionic intermediate in $Z \mathbf{1}-Z \mathbf{6}$ being $>8 \mathrm{kcal} \mathrm{mol}^{-1}$.

The differential rate law for reduction of disulphides 1-6 is given by equations ( 1 and 2). To estimate the rate constants for the formation of the strain-free zwitterionic intermediates (Fig. 3b), we measured the reduction kinetics of disulphides $Z \mathbf{1}-Z \mathbf{6}$ in the presence of triflic acid $(100 \mu \mathrm{M}-3 \mathrm{mM})$. As predicted by equation (2), the reduction accelerates with $\mathrm{H}^{+}$ concentration (Supplementary Tables S5 and S6). Least-square fitting of measured bimolecular rate constants, $k^{Z}$, to equation (2) yielded rate constants for the formation of the zwitterionic intermediate, $k_{1}^{Z}$ (Table 1). These values are similar to those reported for reduction of simple alkyl disulfides by phenyl phosphines under strong basic conditions, where the formation of the RS- $\mathrm{PR}_{3}^{\prime+}$ intermediate is thought to be rate limiting. ${ }^{51}$ Consistent with the mechanism in Fig. $3 \mathrm{~b},\left(k_{1}^{Z} / k^{Z}-1\right)^{-1}$ versus $\left[\mathrm{H}^{+}\right]$correlations were linear (Fig. 5). The variation of $k_{1}^{Z}$ values across disulphides $Z \mathbf{1}-Z \mathbf{6}$ probably reflects the electronic influence of the linkers connecting the disulphide moiety to stiff stilbene (red, Fig. 3b).

$$
\begin{gathered}
-\frac{\mathrm{d}[\mathrm{RSSR}]}{\mathrm{d} t}=k \frac{\left[\mathrm{PR}^{\prime}\right]_{\mathrm{o}}}{1+K_{\mathrm{P}}\left[\mathrm{H}^{+}\right]_{\mathrm{o}}}[\mathrm{RSSR}]_{\mathrm{o}} \\
k=\frac{k_{1}\left(k_{2}+K_{\mathrm{s}}\left[\mathrm{H}^{+}\right]_{\mathrm{o}} k_{2}^{\prime}\right)}{k_{-1}+k_{2}+K_{\mathrm{s}}\left[\mathrm{H}^{+}\right]_{\mathrm{o}} k_{2}^{\prime}}
\end{gathered}
$$

To study force-dependent kinetics of disulphide reduction by phosphines without the complications inherent to polymers and micromanipulation techniques we used the $E$ isomers of disulphides 1-6 (Fig. 3b). The molecular strap containing the disulphide moiety, which bridges the two phenyl rings of stiff stilbene is too short to accommodate the strain-free $E$ geometry of the central $\mathrm{C}=\mathrm{C}$ bond. Consequently, photoisomerization of $Z$ stiff stilbene in $Z \mathbf{1}-Z \mathbf{6}$ creates strained analogues in which the distribution of kinetically significant molecular strain within the reactive moiety approximates that of the same moiety in a stretched oligomer. ${ }^{3}$ Photostationary states containing up to $50 \%$ of strained disulphides $E \mathbf{1}-E \mathbf{6}$ were obtained by irradiation of $\sim 20 \mu \mathrm{M}$ solutions of the $\mathrm{Z}$ analogues in $\mathrm{CH}_{3} \mathrm{CN}$ under rigorously anhydrous and anaerobic conditions (Supplementary Table S2). Most kinetic measurements were conducted on these photostationary states to minimize the measurement error of the ratio of the rate constants, $k^{\mathrm{E} / k^{Z}}$ (see below). Other conditions were identical to those used for $Z \mathbf{1}-Z \mathbf{6}$. Table 1 lists the measured rate constants in the absence of added $\left[\mathrm{H}^{+}\right]$. Unlike the reduction of the $Z$ disulphides, triflic acid did not affect the apparent bimolecular rate constants for the reduction of the $E$ analogues (Supplementary Table S8). 
a

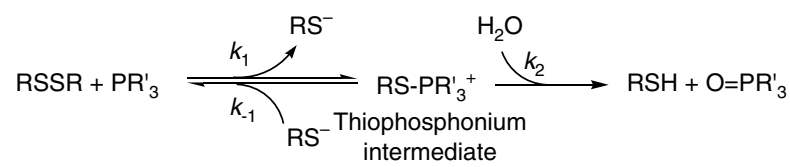

Acid-base equilibria:

$$
\mathrm{RS}^{-}+\mathrm{H}^{+} \stackrel{K_{\mathrm{s}}}{\rightleftharpoons} \mathrm{RSH} \quad \mathrm{PR}_{3}^{\prime}+\mathrm{H}^{+} \stackrel{K_{\mathrm{p}}}{\rightleftharpoons} \mathrm{HPR}_{3}{ }^{+}
$$

b

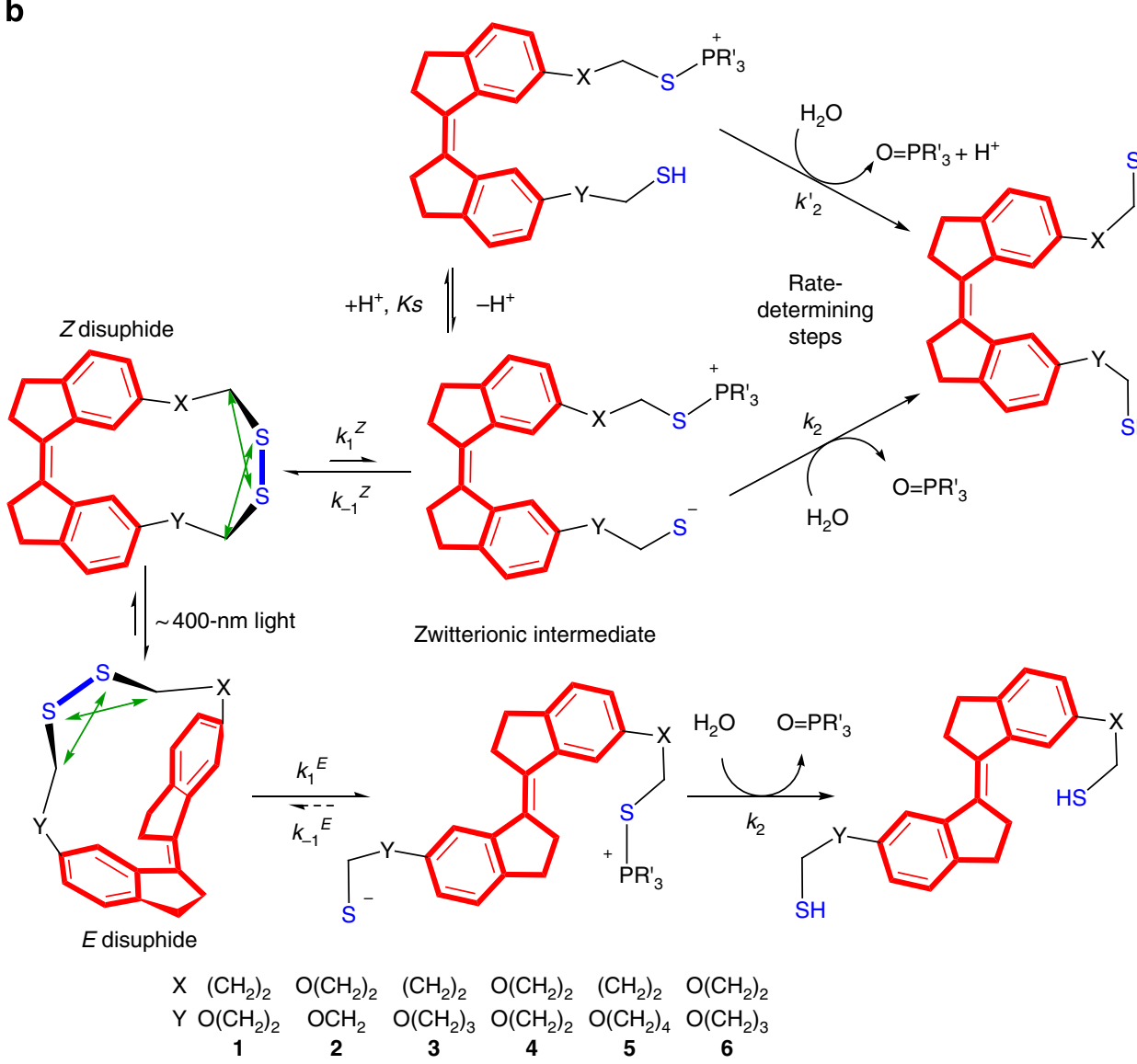

Figure 3 | The mechanism of reduction of disulphides by a phospine in $\mathbf{H}_{\mathbf{2}} \mathbf{O}$. (a) Acyclic disulphides. (b) A series of strain-free ( $Z$ ) and increasingly strained $(E)$ cyclic disulphides used to study the effect of stretching force on the kinetics and mechanism of a pre-equilibrium reaction. The strain of the disulphide moiety in the macrocycles was quantified as the restoring force of the two $\mathrm{H}_{2} \mathrm{C} \cdots \mathrm{S}$ local coordinates (green arrows). Restoring force that develops in an elastically deformed object, opposes further deformation and is responsible for the recovery of the original shape after load removal. In the harmonic approximation, the molecular compliance matrix relates the restoring force of a local coordinate of a molecule both to the absolute distortion of this coordinate and to the externally imposed force. The restoring force is rigorously defined both at the continuum and atomistic levels, ${ }^{3}$ connecting the two descriptions of the response of an object to load. It is particularly valuable in describing the reaction kinetics of stretched polymers, where partial relaxation of non-reactive molecular degrees of freedom far removed from the reactive site makes large contributions to activation barriers. The restoring force provides a simple estimate of this contribution without treating such molecular coordinates explicitly.

DFT calculations. To use the measured data to test the model of the kinetics of double-barrier reaction under stretching force (Fig. 2) we first optimized all thermally accessible conformers of both isomers of disulphides 1-6 as well as the transition states for the formation of the zwitterionic intermediates (Fig. 3b) at the B3LYP/6-311 + G(d) level of the DFT with $\mathrm{H}_{2} \mathrm{O}$-parameterized SMD model ${ }^{53}$ as a continuum representation of the reaction solvent (Supplementary Tables S9 and S10 list the key parameters of the reactant and transition states and Supplementary Tables S11-S13 list the parameters of individual conformers). These calculations yielded free energies of activation of the first step in a good agreement with the measured ( $E$ isomers) or fitted
( $Z$ isomers) values (Table 2: the mean absolute deviation of $1.3 \mathrm{kcal} \mathrm{mol}^{-1}$ ).

We quantified the strain of the disulphide moiety in each macrocycle as the ensemble-average restoring force of the non-bonding $\mathrm{H}_{2} \mathrm{C} \cdots \mathrm{S}$ distances (green arrows, Fig. 3b), which we previously showed to be a useful 1-dimensional representation of the full reaction coordinate of $\mathrm{S}_{\mathrm{N}} 2$ displacement at $\mathrm{S}^{29}$ We obtained the restoring forces by comparing the ensemble-average $\mathrm{H}_{2} \mathrm{C} \cdots \mathrm{S}$ distances in each macrocycle against the calibration curve relating the equivalent ensembleaverage distances and their restoring forces in $\mathrm{Et}_{2} \mathrm{~S}_{2}$ with an external stretching force applied across the terminal $\mathrm{C}$ atoms 


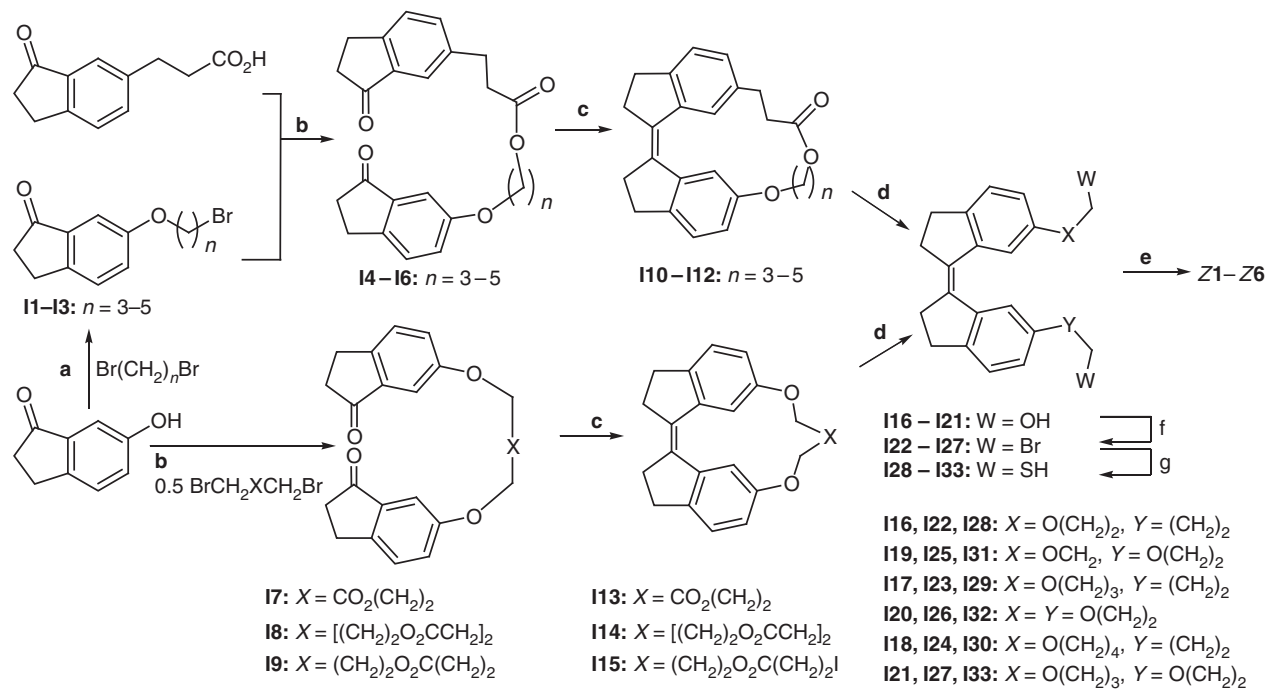

Figure 4 | Synthesis of macrocyclic disulphides Z1-Z6. Summary of synthetic conditions: (a) $\mathrm{Bu}_{4} \mathrm{NBr}_{1} \mathrm{~K}_{2} \mathrm{CO}_{3}$, acetone, reflux; (b) $\mathrm{Bu}{ }_{4} \mathrm{NBr}, \mathrm{K}_{2} \mathrm{CO}_{3}$, $\mathrm{DMF}$, $65^{\circ} \mathrm{C}$; (c) $\mathrm{TiCl}_{4}, \mathrm{Zn}$, THF, reflux; (d) $\mathrm{LiAlH}_{4}$, THF, reflux; (e) I $\mathrm{I}_{2} \mathrm{NEt}_{3}, \mathrm{CHCl}_{3}, 25^{\circ} \mathrm{C}$; (f) $\mathrm{CBr}_{4}, \mathrm{PPh}_{3}$, acetone, $\mathrm{CH}_{2} \mathrm{Cl}_{2}, 25^{\circ} \mathrm{C}$; $(\mathbf{g})$ thiourea, $\mathrm{DMF}, 80^{\circ} \mathrm{C}$, then $\mathrm{NaOH}, 25^{\circ} \mathrm{C}$, then $\mathrm{HCl}, 25^{\circ} \mathrm{C}$. See the Methods section for the synthetic protocols and Supplementary Tables S1 and S2 for the spectroscopic characterization and reaction yields.

Table 1 | Measured reduction kinetics of disulphides 1-6.

\begin{tabular}{|c|c|c|c|c|c|c|}
\hline & \multicolumn{2}{|c|}{$k_{1}^{Z}, M^{-1} s^{-1}$, fitted } & \multicolumn{2}{|c|}{$k^{E}, M^{-1} s^{-1}$, measured } & \multicolumn{2}{|c|}{$\ln \left(k^{E} / k_{1}^{Z}\right)$} \\
\hline & $\mathrm{PPh}_{2} \mathrm{CH}_{3}$ & $\mathbf{P P h}_{3}$ & $\mathrm{PPh}_{2} \mathrm{CH}_{3}$ & $\mathbf{P P h}_{\mathbf{3}}$ & $\mathrm{PPh}_{2} \mathrm{CH}_{3}$ & $\mathbf{P P h}_{3}$ \\
\hline 1 & $3.1 \pm 0.4$ & $0.21 \pm 0.03$ & $6.54 \pm 0.15$ & $0.536 \pm 0.014$ & $0.75 \pm 0.13$ & $0.94 \pm 0.15$ \\
\hline 2 & $6.0 \pm 0.5$ & $0.35 \pm 0.05$ & $17.1 \pm 0.5$ & $1.38 \pm 0.07$ & $1.04 \pm 0.09$ & $1.37 \pm 0.15$ \\
\hline 3 & $11.4 \pm 0.4$ & $1.5 \pm 0.3$ & $3.40 \pm 0.08$ & $0.224 \pm 0.015$ & $-1.21 \pm 0.04$ & $-1.9 \pm 0.2$ \\
\hline 4 & $5.8 \pm 0.5$ & $0.66 \pm 0.13$ & $8.20 \pm 0.11$ & $0.836 \pm 0.011$ & $0.35 \pm 0.09$ & $0.24 \pm 0.19$ \\
\hline 5 & $5.5 \pm 0.6$ & $0.65 \pm 0.12$ & $1.91 \pm 0.13$ & $0.145 \pm 0.015$ & $-1.06 \pm 0.13$ & $-1.5 \pm 0.2$ \\
\hline 6 & $4.4 \pm 0.5$ & $0.30 \pm 0.05$ & $5.05 \pm 0.09$ & $0.472 \pm 0.016$ & $0.14 \pm 0.12$ & $0.45 \pm 0.17$ \\
\hline
\end{tabular}

(Fig. 6), obtained computationally as described in the Methods section. ${ }^{25,32}$

\section{Discussion}

The measured reduction rate constant of an $E$ disulphide, $k^{\mathrm{E}}$, normalized to the extrapolated rate constant for the formation of the zwitterionic intermediate in the corresponding $Z$ analogue $\left(k_{1}^{Z}\right)$, $\ln \left(k^{E} / k_{1}^{Z}\right)$ are plotted in Fig. $7 \mathrm{a}$ against the restoring force of the local coordinate (green arrows, Fig. $3 b$ ), in the $E$ reactant, $\left\langle f_{1}^{E}\right\rangle$. We analyse the $\ln \left(k^{E} / k_{1}^{Z}\right)$ ratio to eliminate rate variations due to the distinct stereoelectronic contributions of different linkers across the series of macrocycles. In our method, different linkers are necessary to control the force exerted on the reactive site, but they may introduce rate variability among different macrocycles that is unrelated to strain. When force on a reactive site can be varied without changing the chemical composition in its vicinity (for example, in single-molecule force experiments on polymers), such normalization of rate constants becomes unnecessary.

The two distinct kinetic regimes (at $\left\langle f_{1}^{E}\right\rangle$ below and above $\sim 120 \mathrm{pN}$ ) apparent across the series of the disulphides (Fig. 7a) are consistent with the simple picture in Fig. 2 . At low forces of weakly strained disulphides E3 and E5 the reduction is accelerated without a change in the rate-determining step (cf. green trace, Fig. 2), as evidenced by the observation that the apparent rate constants of reduction of $E 3$ and $E 5$ exceeds that of their strain-free analogues, $Z 3$ and $Z 5$, but remains smaller than that of the first reduction step, that is, the formation of the strain-free zwitterionic intermediate (that is, $k^{Z}<k^{E}<k_{1}^{Z}$, for example, $0.19 \pm 0.04,1.91 \pm 0.19$ and $5.5 \pm 0.6 \mathrm{M}^{-1} \mathrm{~s}^{-1}$, respectively, for 5 ).

At higher forces (disulphides E1,E2, E4, and E6), the apparent reduction rate constants $k^{E}$, both exceed those of the formation of the zwitterionic intermediate of the corresponding strain-free $Z$ analogues, and are independent of the solution acidity (Supplementary Table S8), suggesting the shift of the ratedetermining step to the previously inner barrier ( $c f$. red line, Fig. 2). Indeed, the slopes of the linear regression of the $\ln \left(k^{E} / k_{1}^{Z}\right)$ vs. $\left\langle f_{1}^{E}\right\rangle$ data above $120 \mathrm{pN}$ (broken lines in Fig. 7a), normalized to thermal energy $(0.25$ and $0.35 \AA$ for the reduction by $\mathrm{PPh}_{2} \mathrm{CH}_{3}$ and $\mathrm{PPh}_{3}$, respectively) are reasonably close to the average elongation of the $\mathrm{H}_{2} \mathrm{C} \cdots \mathrm{S}$ separation from the reactant to the transition state in macrocyclic disulphides $(0.16$ and $0.24 \AA$, Supplementary Tables S9 and S10; Supplementary Figs. S1 and S2), in accord with the simplest model of singlebarrier force-dependent kinetics. ${ }^{54}$

To confirm the conclusion that reduction of disulphides $E \mathbf{1}$, $E 2, E 4$, and $E 6$ is limited by the force-dependent kinetics of the formation of the zwitterionic intermediate, rather than the force-independent hydrolysis of the zwitterionic intermediate, as in the strain-free disulphide, we calculated (at the B3LYP/6- 
$311+\mathrm{G}(\mathrm{d})$ level in $\mathrm{H}_{2} \mathrm{O}$-parameterized $\mathrm{SMD}^{53}$ ) the activation free energy of the first step in reduction of $\mathrm{Et}_{2} \mathrm{~S}_{2}$ by $\mathrm{PPh}_{3}$ and $\mathrm{PPh}_{2} \mathrm{CH}_{3}$ (Fig. 3a) as a function of the stretching force applied across the terminal $\mathrm{C}$ atoms of $\mathrm{Et}_{2} \mathrm{~S}_{2}$, using previously described methodology. ${ }^{25,32}$ Stretching force lowers the first barrier for both phosphines as indicated by positive values of $\ln \left[k_{1}(f) / k_{1}^{o}\right]$, where $k_{1}^{\mathrm{o}}$ is the rate constant in the absence of force. The measured values of $\ln \left(k^{E} / k_{1}^{Z}\right)$ for disulphides $1,2,4,6$ follow this trend closely, suggesting that the acceleration of reduction of $E \mathbf{1}, E 2, E 4$ and $E \mathbf{6}$ relative to their strain-free $Z$ analogues can be attributed to force-dependent lowering of the first (now rate-determining)
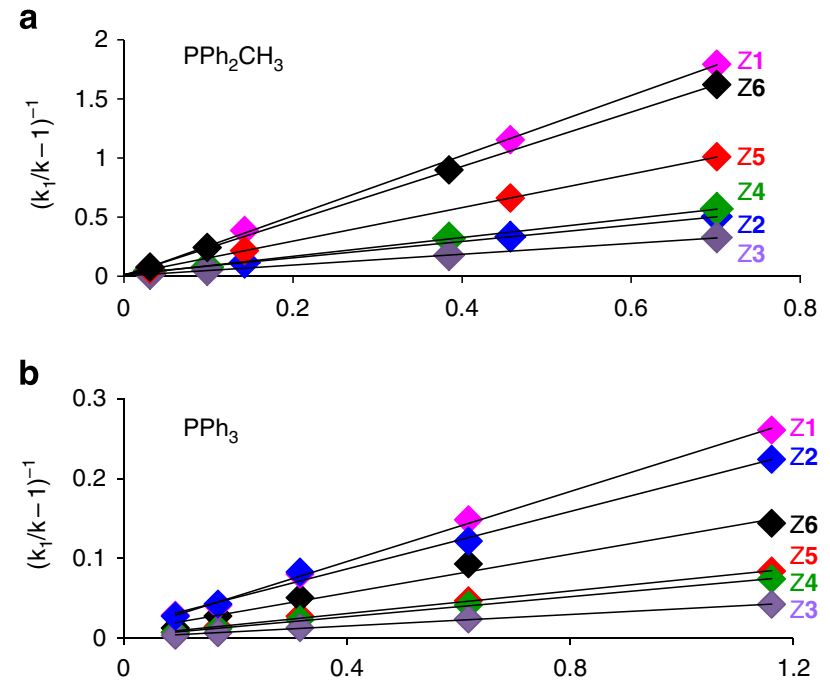

$\left[\mathrm{H}^{+}\right], \mathrm{mM}$

Figure 5 | Acceleration of the reduction of strain-free disulphides Z1-Z6 in acidic solution. Linearity of $\left(k_{1}^{Z} / k^{Z}-1\right)^{-1}$ versus $\left[\mathrm{H}^{+}\right]$graphs (a: by $\mathrm{PPh}_{2} \mathrm{Me}$; b: by $\mathrm{PPh}_{3}$ ) is consistent with reduction of disulphides $Z \mathbf{1}-Z \mathbf{6}$ proceeding by the mechanism in Fig. 3b. Lines are linear least-squares fits. The intercepts $\left(k_{2} / k_{-1}\right)$ range from $(1.7 \pm 0.2) \times 10^{-2}$ to $(1.1 \pm 0.5) \times 10^{-3}$, indicating that under neutral conditions, a strain-free zwitterionic intermediate is 60 to $10^{3}$ times more likely to revert back to the disulphide than to undergo hydrolysis to the products, again consistent with the postulated reaction mechanism. The intercept-to-slope ratios $\left(K_{s} k_{2}^{\prime} / k_{2}\right)$ averaged $\sim 3 \mu \mathrm{M}$ for reduction with $\mathrm{PPh}_{2} \mathrm{CH}_{3}$ and $\sim 40 \mu \mathrm{M}$ for reduction with $\mathrm{PPh}_{3}$ and equal proton concentration, $\left[\mathrm{H}^{+}\right]$, at which half of the reaction proceeds through the protonated intermediate. The listed values are averages of three independent measurements; s.d. are smaller than the size of the markers. The plotted data is tabulated in Supplementary Tables S5-S7. barrier. The deviation of the measured $\ln \left(k^{E} / k_{1}^{Z}\right)$ ratio for disulphides 3 and 5 from the computed $\ln \left(k_{1}\left(f_{1}\right) / k_{1}^{\circ}\right)$ correlation is consistent with the rate-determining step in these weakly strained disulphides being the same as in the absence of strain, that is, hydrolysis of the zwitterionic intermediate (Fig. 3b).

We conclude that, at least for the reduction of a disulphide by a phosphine, the simple model of force-dependent kinetics of a multi-barrier reaction shown in Fig. 2 is valid. Although the second step, which is rate-determining at low-stretching forces, is orthogonal to the pulling axis and is therefore not affected by force, the overall reaction is accelerated by force because force suppresses reversion of the intermediate to the reactant. The force-rate correlation shows two kinetic regimes and a usefully accurate estimate of the rate constant for the formation of strainfree intermediate is obtained from extrapolating the high-force regime to zero force. The accuracy of the extrapolated strain-free 'inner' barrier height (or the corresponding rate constant) depends strongly on identifying the minimum force corresponding to the change in the rate-determining step and is thus facilitated by the fairly sharp delineation of the two kinetic regimes in Fig. 7a. The existence of multiple energy barriers preceding the rate-determining transition states may obscure such turnover regimes, potentially complicating the molecular interpretation of rate-force correlations. Distinct kinetic regimes at small and large forces are sometimes observed in singlemolecule force experiments ${ }^{43}$ but whether they represent changes

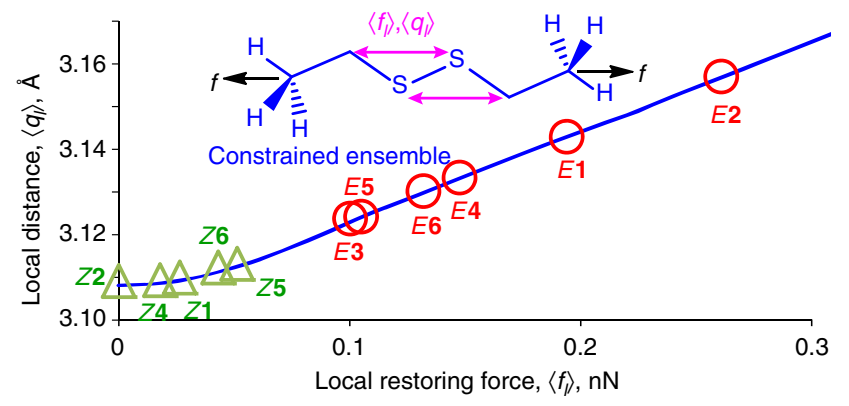

Figure 6 | The calculated distance-force correlation of the $\mathrm{H}_{2} \mathrm{C} \cdots \mathrm{S}$ coordinate of aliphatic disulphide. The restoring forces of the disulphide moieties in 1-6, $\left\langle f_{1}\right\rangle$, were obtained by comparing computed $\mathrm{H}_{2} \mathrm{C} \cdots \mathrm{S}$ separations, $\left\langle q_{1}\right\rangle$, in disulphides 1-6 (green arrows in Fig. $3 b$ ) to the forceextension curve for the ensemble of $\mathrm{Et}_{2} \mathrm{~S}_{2}$ with the stretching force applied across the terminal $C$ atoms. All calculations were at the B3LYP/6$311+\mathrm{G}(\mathrm{d})$ level in SMD. In $Z \mathbf{3}\left\langle q_{1}\right\rangle=3.106 \AA$, corresponding to a compressive force of $35 \mathrm{pN}$ and is not shown on the graph. The plotted data is tabulated in Supplementary Tables S9 and S14.

Table 2 | Activation free energies of disulphide reduction.

\begin{tabular}{|c|c|c|c|c|c|c|c|c|}
\hline & \multicolumn{4}{|c|}{ Experiment } & \multicolumn{4}{|c|}{ B3LYP/6-311 + G(d) $/$ SMD $^{\star}$} \\
\hline & \multicolumn{2}{|c|}{$\mathbf{P P h}_{\mathbf{3}}$} & \multicolumn{2}{|c|}{$\mathrm{PPh}_{2} \mathrm{CH}_{3}$} & \multicolumn{2}{|c|}{$\mathbf{P P h}_{\mathbf{3}}$} & \multicolumn{2}{|c|}{$\mathrm{PPh}_{2} \mathrm{CH}_{3}$} \\
\hline & $\mathbf{Z}$ & $\mathbf{E}$ & $\mathbf{Z}$ & $\mathbf{E}$ & $\mathbf{Z}$ & $\mathbf{E}$ & $\mathbf{Z}$ & $\mathbf{E}$ \\
\hline 1 & $18.6 \pm 0.1$ & $18.0 \pm 0.1$ & $17.0 \pm 0.1$ & $16.8 \pm 0.1$ & 19.8 & 19.4 & 18.1 & 18.2 \\
\hline 2 & $18.3 \pm 0.1$ & $17.5 \pm 0.1$ & $16.6 \pm 0.1$ & $16.0 \pm 0.1$ & 20.0 & 19.0 & 17.9 & 15.9 \\
\hline 3 & $17.4 \pm 0.1$ & $18.6 \pm 0.1$ & $16.2 \pm 0.1$ & $16.9 \pm 0.1$ & 15.7 & 19.6 & 16.7 & 18.3 \\
\hline 4 & $17.9 \pm 0.1$ & $17.8 \pm 0.1$ & $16.6 \pm 0.1$ & $16.4 \pm 0.1$ & 18.8 & 18.6 & 17.9 & 16.9 \\
\hline 5 & $17.9 \pm 0.1$ & $18.8 \pm 0.1$ & $16.7 \pm 0.1$ & $17.3 \pm 0.1$ & 19.5 & 19.7 & 15.0 & 16.6 \\
\hline 6 & $18.4 \pm 0.1$ & $18.1 \pm 0.1$ & $16.8 \pm 0.1$ & $16.7 \pm 0.1$ & 20.8 & 19.5 & 17.5 & 16.8 \\
\hline
\end{tabular}




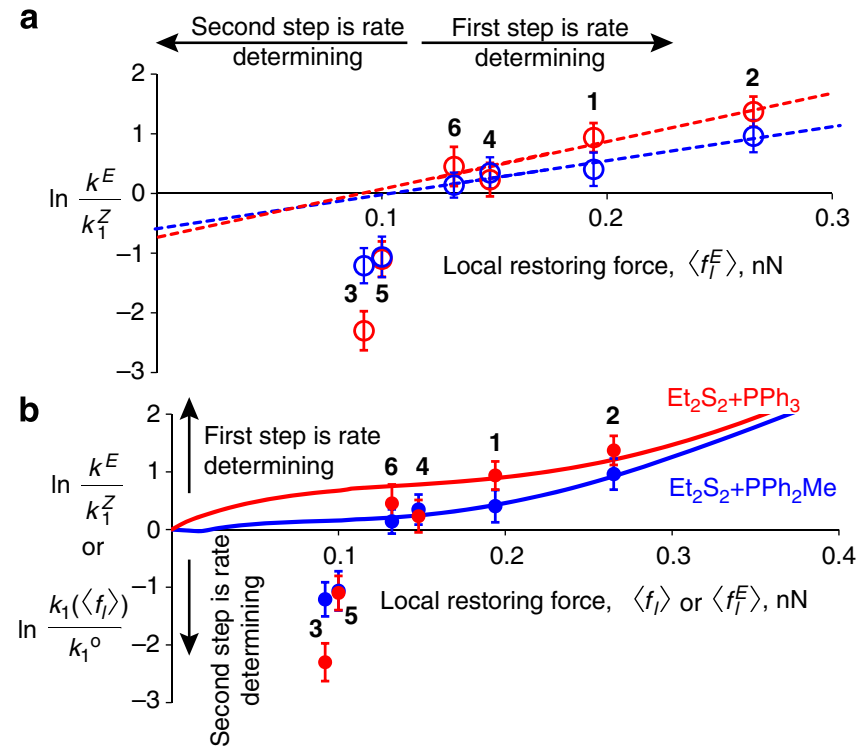

Figure 7 | Reduction kinetics as a function of the force applied to the disulphide moiety. (a). Log of the ratio of the reduction rate constants of strained disulphides $E \mathbf{1}-E \mathbf{6}$ to the rate constants of the formation of the zwiterionic intermediate in strain-free $\mathbf{Z 1}-Z \mathbf{6}$ analogues with $\mathrm{PPh}_{3}$ (red) and $\mathrm{PPh}_{2} \mathrm{CH}_{3}$ (blue) versus the ensemble-average restoring force of the $\mathrm{H}_{2} \mathrm{C} \cdots \mathrm{S}$ coordinate of the disulphide moiety (green arrows, Fig. $3 b$ ) in E1-E6, $\left\langle f_{\uparrow}^{E}\right\rangle$. Broken lines are linear least-squares fits to data for disulphides $\mathbf{1}, \mathbf{2}, \mathbf{4}$, and 6. (b) The calculated acceleration of the first step of disulphide reduction (formation of the thiophosphonium intermediate, solid lines) and measured acceleration of reduction of disulphides 1-6 (points) versus local restoring force. As described elsewhere in the manuscript, the deviation of the measured $\ln \left(k^{E} / k_{1}^{Z}\right)$ ratio for disulphides $\mathbf{3}$ and $\mathbf{5}$ from the computed $\ln \left(k_{1}\left(\left\langle f_{1}\right\rangle\right) / k_{1}^{\circ}\right)$ values (solid lines) is consistent with the stretching force applied to the disulphide moiety in E3 and E5 being too small to shift the rate-determining step to the formation of the zwitterionic intermediate (that is, similar to the green line in Fig. 2). The error bars reflect the standard deviations of three independent measurements. The nonlinearity of $\Delta \Delta G_{1}^{\ddagger} \propto \ln \left(k_{1}\left(\left\langle f_{1}\right\rangle\right) / k_{1}^{\circ}\right)$ versus $f$ correlations (solid lines) results primarily from the strong dependence of the difference in the constrained distance between the reactant and the transition state (Supplementary Figs S1 and S2). The plotted data are tabulated in Table 1 and Supplementary Table S14. The $\Delta G_{1}^{\ddagger}\left(\left\langle f_{1}\right\rangle\right)$ correlations up to $1.5 \mathrm{nN}$ are shown in Supplementary Fig. S3.

in the rate-determining step remains uncertain. ${ }^{45,55}$ Applying the methodology described above to other reactions that traverse multiple barriers (for example, those in Fig. 1) would help define the limits of applicability of the simple picture in Fig. 2.

\section{Methods}

General. Structures of synthetic intermediates I3-I33 are shown in Figure 4; the reaction yields and spectroscopic characterization are in Supplementary Tables S1 and S2. The disulphides 1-6 and the diols I28-I33 were first reported in Kucharski et al..$^{29}$ Commercial reagents of the highest available purity from Aldrich or Fisher were used without further purification unless stated otherwise. Water was obtained from a Milli-Q water purification system. Analytical and preparative thin-layer chromatography was performed on silica gel 60 from Fisher and Aldrich. The concentrations of aqueous trifluoromethanesulphonic acid (TfOH) solutions were determined by titration with commercial standards.

Irradiation was performed in vessels sealed under $\mathrm{N}_{2}$ using high-intensity diode light sources from Opto Technology with light output at $375 \pm 7 \mathrm{~nm}$. Light intensity was controlled with a custom-made constant-current controller. UV-vis absorption spectra were recorded on a Cary 50 UV-vis spectrophotometer. The diode light source was oriented $90^{\circ}$ from the spectrophotometer beam path so that UV-vis absorbance spectra could be measured during irradiation to monitor reaction progress.
Analytical HPLC was performed on a Shimadzu Prominence LC system with LC-20AT solvent delivery unit, DGU-20A5 degasser, SPD-M20A photodiode array detector, CBM-20A system controller and Rheodyne 7725i manual injector, with either a J. T. Baker C18 column $(25 \mathrm{~cm} \times 4.6 \mathrm{~mm}, 5 \mu \mathrm{m}$ particles $)$, Supelco C18 column $(15 \mathrm{~cm} \times 4.6 \mathrm{~mm}, 5 \mu \mathrm{m}$ particles), or Agilent Hypersil AA-ODS (C18) column $(20 \mathrm{~cm} \times 2.1 \mathrm{~mm}, 5 \mu \mathrm{m}$ particles $)$.

High-resolution mass spectrometry (HRMS) was performed on a Micromass 70-VSE mass spectrometer (EI) or a Waters Q-Tof Ultima mass spectrometer (ESI) at the University of Illinois Mass Spectrometry Center. NMR spectra of macrocycles 1-6 and synthetic intermediates were recorded on 400 Unity-INOVA Varian spectrometers at room temperature $\left(\sim 20^{\circ} \mathrm{C}\right)$. Chemical shifts are reported in p.p.m. downfield from that of tetramethylsilane and are referenced to the residual ${ }^{1} \mathrm{H}$ resonances of the deuterated solvents. ${ }^{31} \mathrm{P}\{1 \mathrm{H}\}$ resonances are reported in p.p.m. downfield from $85 \% \mathrm{H}_{3} \mathrm{PO}_{4}$, but were not externally referenced.

Synthesis of I1-I3. 6-Hydroxyindanone ( $0.3 \mathrm{~g}), \mathrm{K}_{2} \mathrm{CO}_{3}$ (1.5 eq.), $\mathrm{N}\left(n-\mathrm{C}_{4} \mathrm{H}_{9}\right)_{4} \mathrm{Br}$ (0.1 eq.) and $\operatorname{Br}\left(\mathrm{CH}_{2}\right)_{n} \mathrm{Br}(n=3,4$ or 5 for $\mathbf{I} 1$, $\mathbf{I} 2$ or $\mathbf{I 3}$, respectively, 6.0 eq.) in acetone $(20 \mathrm{ml})$ were refluxed for $\sim 16 \mathrm{~h}$, cooled to room temperature (RT), and poured in $\mathrm{H}_{2} \mathrm{O}(60 \mathrm{ml})$. The product was extracted with $\mathrm{CH}_{2} \mathrm{Cl}_{2}(3 \times 40 \mathrm{ml})$. The organic fractions were dried over $\mathrm{MgSO}_{4}$, filtered and concentrated under reduced pressure. The crude product was purified by flash chromatography with hexanes followed by $\mathrm{CH}_{2} \mathrm{Cl}_{2}$ to afford I1-I3 as pale yellow solids.

Synthesis of 14-16. 3-(6-Indan-1-one)propionic acid ( $0.3 \mathrm{~g}), \mathrm{K}_{2} \mathrm{CO}_{3}$ (1.5 eq.), I1, I2 or I3 (1.0 eq.), and $\mathrm{N}\left(n-\mathrm{C}_{4} \mathrm{H}_{9}\right)_{4} \mathrm{Br}(0.1$ eq.) in dry DMF $(20 \mathrm{ml})$ were heated at $65^{\circ} \mathrm{C}$ for $\sim 20 \mathrm{~h}$. The mixture was cooled to RT, diluted with $\mathrm{CH}_{2} \mathrm{Cl}_{2}(100 \mathrm{ml})$ and washed with brine $(4 \times 100 \mathrm{ml})$. The organic fractions were dried over $\mathrm{MgSO}_{4}$, filtered and concentrated under vacuum to afford I4-I6 as light-brown solids.

Synthesis of 17-19. 3-bromopropyl 2-bromoacetate (for I7), bis(3-bromopropyl) succinate (for I8), or 3-bromopropyl 4-bromobutanoate (for I9) (0.5 eq.), 6hydroxyindanone ( $0.70 \mathrm{~g}, 1$ eq.), $\mathrm{N}\left(n-\mathrm{C}_{4} \mathrm{H}_{9}\right)_{4} \mathrm{Br}$ ( 0.1 eq.), and $\mathrm{K}_{2} \mathrm{CO}_{3}$ (2 eq.) in dry DMF $(20 \mathrm{ml})$ were stirred at $65{ }^{\circ} \mathrm{C}$ overnight under $\mathrm{N}_{2}$. Upon cooling to RT, dry $\mathrm{CH}_{2} \mathrm{Cl}_{2}(50 \mathrm{ml})$ was added, solids were filtered off and the filtrate was washed with $\mathrm{H}_{2} \mathrm{O}(3 \times 100 \mathrm{ml})$. The organic fractions were dried over $\mathrm{MgSO}_{4}$, filtered and dried under reduced pressure to afford $\mathbf{I 7 - I 9}$ as brown solids.

McMurry coupling. $\mathrm{TiCl}_{4}(6$ eq.) was added to a stirred suspension of $\mathrm{Zn}$ powder (12 eq.) in dry THF $(\sim 50 \mathrm{ml})$ over $10 \mathrm{~min}$ at $0^{\circ} \mathrm{C}$. The resulting slurry was refluxed for $1.5 \mathrm{~h}$. To the refluxing mixture $\mathbf{I 4}, \mathbf{I 5}, \mathbf{I 6}, \mathbf{I 7}$, I8 or $\mathbf{I 9}(0.6 \mathrm{~g}, 1 \mathrm{eq}$.) in THF $(\sim 100 \mathrm{ml})$ was added over $\sim 5 \mathrm{~h}$ by syringe pump. The reflux was continued for $20-40 \mathrm{~min}$. The mixture was cooled to RT, poured into a saturated aqueous solution of $\mathrm{NH}_{4} \mathrm{Cl}$ and the product was extracted with $\mathrm{CH}_{2} \mathrm{Cl}_{2}$. The organic fractions were dried over $\mathrm{MgSO}_{4}$, and I10-I15 were obtained as yellow solids after solvent removal.

Reduction of esters I10-I15 to diols I16-I21. $\mathrm{LiAlH}_{4}$ (2 eq.) was added to a stirred solution of I10-I15 ( $0.4 \mathrm{~g}, 1$ eq.) in dry THF $(\sim 40 \mathrm{ml})$ under $\mathrm{N}_{2}$. The resulting mixture was refluxed for $4 \mathrm{~h}$. After cooling to RT, the reaction was quenched with $\mathrm{H}_{2} \mathrm{O}$ and the solids were filtered off. The filtrate was evaporated, dissolved in $\mathrm{CH}_{2} \mathrm{Cl}_{2}$, washed with $\mathrm{H}_{2} \mathrm{O}$ and dried over $\mathrm{MgSO}_{4}$. The product (I16I21) obtained after $\mathrm{CH}_{2} \mathrm{Cl}_{2}$ removal was used without purification.

Bromination of diols I16-I21 to dibromides I22-I27. $\mathrm{PPh}_{3}$ (4 eq.) was added carefully to a stirred solution of $\mathbf{I 1 6}-\mathbf{I 2 1}\left(0.3 \mathrm{~g}, 1\right.$ eq.) and $\mathrm{CBr}_{4}$ (4 eq.) in $\mathrm{CH}_{2} \mathrm{Cl}_{2}$ $(20 \mathrm{ml})$ containing five drops of acetone. The resulting mixture was stirred for $10-$ $15 \mathrm{~min}$ and purified immediately by flash chromatography on silica with a gradient from hexanes to $\mathrm{CH}_{2} \mathrm{Cl}_{2}$. The product was further purified with preparative centrifugally accelerated radial thin-layer chromatography to afford pure I22-I27 as yellow oils.

Synthesis of Z1-Z6. Thiourea (4 eq.) and dibromides I22-I27 ( $0.2 \mathrm{~g}, 1$ eq.) in DMF $(20 \mathrm{ml})$ were stirred at $80^{\circ} \mathrm{C}$ under $\mathrm{N}_{2}$ overnight. After cooling to RT, degassed $1 \mathrm{M} \mathrm{NaOH}$ aqueous solution $(20 \mathrm{ml})$ was added under $\mathrm{N}_{2}$, the mixture was stirred at RT for $50 \mathrm{~min}$, acidified with degassed $1 \mathrm{M} \mathrm{HCl}$ to $\mathrm{pH}=3$ and the product was extracted with $\mathrm{CH}_{2} \mathrm{Cl}_{2}$. Drying with $\mathrm{MgSO}_{4}$, filtration and evaporation of $\mathrm{CH}_{2} \mathrm{Cl}_{2}$ afforded dithiols I28-I33, which were oxidized immediately as follows. Solutions of $\mathrm{I}_{2}$ and $\mathbf{I 2 8}-\mathbf{I} 33$ in $\mathrm{CHCl}_{3}$ were added simultaneously by syringe pump at the same rate over $\sim 3 \mathrm{~h}$ to $\mathrm{CHCl}_{3}(50 \mathrm{ml})$ containing $\left.\mathrm{N}_{(} \mathrm{C}_{2} \mathrm{H}_{5}\right)_{3}(1 \mathrm{ml})$. The mixture was poured in $\mathrm{H}_{2} \mathrm{O}$, acidified to $\mathrm{pH}=3$ with $1 \mathrm{M} \mathrm{HCl}$, and unreacted $\mathrm{I}_{2}$ was removed with $\mathrm{Na}_{2} \mathrm{~S}_{2} \mathrm{O}_{3}$. The isolated organic fractions were dried over $\mathrm{MgSO}_{4}$. The crude product was purified with centrifugally accelerated radial thin-layer chromatography eluting with $\mathrm{CH}_{2} \mathrm{Cl}_{2} /$ hexanes $(2: 3 \mathrm{~mol}$ ) to afford pure $\mathbf{Z 1}-\mathbf{Z 6}$.

Kinetic measurements. The reduction kinetics was measured under pseudo-firstorder conditions in phosphine $\left(0.2-1 \mathrm{mM}\right.$ of $\mathrm{PPh}_{2} \mathrm{CH}_{3}$ or $5 \mathrm{mM}$ of $\mathrm{PPh}_{3}$ and $20 \mu \mathrm{M}$ for a disulphide) under rigorously anaerobic conditions. Because in acidic 
solutions, partial protonation of phosphine lowers its effective concentration, we measured the protonation constants of the phosphines, $K_{\mathrm{p}}$, in $\mathrm{H}_{2} \mathrm{O}: \mathrm{CH}_{3} \mathrm{CN}$ (3:1 mol). We extracted $K_{\mathrm{p}}$ from pseudo-first-order reduction rate constants of $E \mathbf{1}, E \mathbf{2}$ and $E \mathbf{6}, k_{\text {pseudo, }}^{E}$ at different concentrations of triflic acid, (TfOH) (Supplementary Table S7), by fitting them to equation (3) $\left(\left[\mathrm{PR}_{3}^{\prime}\right]_{\mathrm{t}}\right.$ is the total initial concentration of the phosphine and $k_{E}$ is the bimolecular rate constant for the phosphine reduction), assuming that the reduction is a single-barrier reaction. The validity of this assumption was confirmed by linearity of the $\left[\mathrm{PR}_{3}^{\prime}\right]_{\mathrm{o}} / k_{\text {pseudo }}^{E}$ versus $\left[\mathrm{H}^{+}\right]$plot (Supplementary Figure $\mathrm{S} 4$ ), where $\left[\mathrm{H}^{+}\right]$was calculated by equation (4). $K_{\mathrm{p}}$ we measured for $\mathrm{PPh}_{3}\left(480 \pm 40 \mathrm{M}^{-1}\right)$ is close to the literature value $\left(\sim 500 \mathrm{M}^{-1}\right)^{49} ; K_{\mathrm{p}}$ for $\mathrm{PPh}_{2} \mathrm{CH}_{3}$ has not been reported.

$$
\begin{aligned}
& k_{\mathrm{pseudo}}^{E}=\frac{k^{E}\left[\mathrm{PR}_{3}^{\prime}\right]_{\mathrm{t}}}{1+\frac{K_{\mathrm{p}}}{2}\left([\mathrm{TfOH}]-K_{\mathrm{p}}^{-1}-\left[\mathrm{PR}_{3}^{\prime}\right]_{\mathrm{t}}+\sqrt{4 K_{\mathrm{p}}^{-1}[\mathrm{TfOH}]+\left(K_{\mathrm{p}}^{-1}-[\mathrm{TfOH}]+\left[\mathrm{PR}_{3}^{\prime}\right]_{\mathrm{t}}\right)^{2}}\right)} \\
& {\left[\mathrm{H}^{+}\right]_{\mathrm{o}}=\frac{1}{2}\left([\mathrm{TfOH}]-K_{\mathrm{p}}^{-1}-\left[\mathrm{PR}_{3}^{\prime}\right]_{\mathrm{o}}+\sqrt{4 K_{\mathrm{p}}^{-1}[\mathrm{TfOH}]+\left(K_{\mathrm{p}}^{-1}-[\mathrm{TfOH}]+\left[\mathrm{PR}_{3}^{\prime}\right]_{\mathrm{o}}\right)^{2}}\right)}
\end{aligned}
$$

Initial guesses for fitting measured $k^{Z}$ rate constants to equation (2) $\left(K_{\mathrm{s}} k_{2}{ }^{\prime} / k_{-1}\right.$ and $\left.k_{2} / k_{-1}\right)$, were obtained by linear least-squares fits (LSFs) of $\left(k^{\mathrm{E}} / 2 k_{-1}\right)^{-1}$ versus $\left[\mathrm{H}^{+}\right]$(that is, by assuming $k_{1}^{\mathrm{Z}}=k^{\mathrm{E}} / 2$ ). The lsqnonlin function of Matlab was used to refine these guesses by non-linear LSFs to equation (2), which yielded more accurate values of $k_{1}^{Z}$ than those underlying the initial guess of the fitting parameters. These new values were used to calculate $\left(k_{1}^{Z} / k_{-1}\right)^{-1}$ and the new values of the fitting parameters $K_{\mathrm{s}} k_{2}^{\prime} / k_{-1}$ and $k_{2} / k_{-1}$ from linear LSFs of $\left(k_{1}^{Z} / k_{-1}\right)^{-1}$ versus $\left[\mathrm{H}^{+}\right]$correlations (that is, similar to Fig. 3 ). The procedure was repeated until the relative change in $k_{1}^{Z}$ between two sequential cycles of lsqnonlin fitting was $<10^{-5}$. The uncertainty of $k_{1}^{Z}$ was estimated from the Jacobian returned by lsqnonlin. ${ }^{56}$ The uncertainties of $K_{s} k_{2}{ }^{\prime} / k_{-1}$ and $k_{2} / k_{-1}$ were estimated from linear LSFs of the $\left(k_{1}^{Z} / k_{-1}\right)^{-1}$ versus $\left[\mathrm{H}^{+}\right]$data using the converged values of $k_{1}^{Z}$ (Supplementary Table S8).

The reduction kinetics of 1-6 was measured on mixtures of different macrocycles, because there was no measureable effect on the reduction kinetics of any individual macrocycle from the presence of other macrocycles in the same reaction mixture. Based on the composition of the mixtures whose constituents could be conveniently separated (Supplementary Table S3), either E-6,6'bismethoxybiindanylidene or ZI10 (Fig. 4) was used as an internal standard. They were chosen because of their availability in high purity, their chemical inertness towards all other components in the reaction mixtures and their retention times on reverse-phase HPLC.

To prevent re-oxidation of the product dithiols by atmospheric oxygen HPLCgrade $\mathrm{CH}_{3} \mathrm{CN}$ and $\mathrm{H}_{2} \mathrm{O}$ used as reaction solvents were thoroughly sparged with $\mathrm{N}_{2}$ before use. ${ }^{1} \mathrm{H}$-NMR spectroscopy confirmed that $\mathrm{CH}_{3} \mathrm{CN}$ solutions of $\mathrm{PPh}_{3}$, and $\mathrm{PPh}_{3} \mathrm{Me}$ were stable for weeks when prepared under these conditions and stored in vials sealed under $\mathrm{N}_{2}$. Reactions were carried out in sealed vials, using a gas-tight syringe to withdraw $\sim 100-\mu \mathrm{l}$ aliquots for composition analysis by HPLC. These samples were transferred to clean vials and either analysed immediately or sealed under $\mathrm{N}_{2}$ and stored at $-35^{\circ} \mathrm{C}$ until analysis.

Reaction vials were held in a custom-made heating block with resistance heaters and a thermocouple connected to an active feedback temperature controller, which enabled the temperature of the reaction mixtures to be maintained with a stability of $\pm 0.1{ }^{\circ} \mathrm{C}$. The reaction temperature was recorded every $6 \mathrm{~s}$. To prevent photoisomerization between $E$ and $Z$ isomers by ambient light, amber glass vials were used and all samples were kept away from light. No changes in $E / Z$ ratios could be detected in samples stored for weeks under these conditions.

Reaction mixtures were prepared by diluting combinations of stock solutions of the disulphides or their photostationary mixtures (for compositions of mixtures, see Supplementary Table S3) with $\mathrm{CH}_{3} \mathrm{CN}$, water, aqueous $\mathrm{TfOH}$, and phosphine in $\mathrm{CH}_{3} \mathrm{CN}$ to give a total absorbance of $\sim 0.3$, corresponding to $\sim 20 \mu \mathrm{M}$ total concentration of disulphide, a total volume of 800 or $1,200 \mu \mathrm{l}$, the desired concentrations of phosphine and $\mathrm{TfOH}$, and $\mathrm{a}_{2} \mathrm{O} / \mathrm{CH}_{3} \mathrm{CN}$ ratio of 3:1 (molar). Reaction mixtures were allowed to equilibrate to the temperature of the heating block before the addition of the phosphine solution. After the addition the reaction vial was shaken vigorously for $\sim 2 \mathrm{~s}$ and returned to the thermostated heating block. Samples were then collected at regular intervals, as described above. Stock solutions of phosphines used were sufficiently concentrated so that the volume of the addition required was minimal $(\sim 10-20 \mu \mathrm{l}$ out of the total $800-1,200 \mu \mathrm{l})$, minimizing cooling of the reaction mixture upon addition.

DFT calculations of the reaction-path data in Fig. 1. All calculations were performed with Gaussian09.B01 and the $6-311+\mathrm{G}(\mathrm{d})$ basis set; dissociation of the anthracene dimer (green) and methylethylidene cyclobutane (blue) were calculated with the uB3LYP functional in the gas phase; the remaining reactions utilized the B3LYP functional using SMD ${ }^{53}$ as a continuum model of reaction solvent $\left(\mathrm{H}_{2} \mathrm{O}\right.$ parameterized SMD for ester and amide bond hydrolysis; methanol-parameterized SMD for degenerate methanolysis of $\left.\mathrm{O}=\mathrm{P}(\mathrm{OMe})_{3}\right)$. Ester and amid hydrolysis used five molecules of microsolvation, as described in Akbulatov et al. ${ }^{48}$ The standard algorithm (Gaussian irc keyword) was used. Reaction-path calculations started from all conformers of the two transition states in both forward and reverse directions. Methylethylidene cyclobutane (blue) and the stationary points along its dissociation path have single conformer. For other reactions, transition states had more conformers than the reactant or the intermediate (that is, multiple conformers of the transition states lead to the same reactant or intermediate). In these cases, only the lowest-energy conformer was considered in ensemble averaging. Several conformers of the intermediate for ester and amide hydrolysis do not appear to be directly linked to either TS1 or TS2, and these conformers were excluded from ensemble averaging.

IRC calculations toward the reactants or the products terminated $2-4 \mathrm{kcal} / \mathrm{mol}$ above the energy of separated reactants, or products, which is typical for bimolecular dissociation reactions. The value of the reaction coordinate corresponding to the reactants was determined by fitting the $\sim 75 \%$ of the computed irc closest to the reactant end to a quadratic function and assigning to the reactants the value of the reaction coordinate corresponding to the minimum of this function. The intrinsic reaction coordinates (in units of bohr $\mathrm{amu}^{0.5}$ ), which were unique for each irc, were rescaled to a uniform scale so that the reactants were assigned a value of -2 ; the TS1, -1 ; the intermediate 0 and the TS2, 1 . The relationship between the intrinsic reaction-coordinate value and the uniform values was fitted to a quadratic equation to position the non-stationary points properly along the new uniform reaction path. On the new reaction-coordinate scale, the irc calculations terminated at the product side at values between 3 and 4.5 (the data plotted in Fig. 1a, main text, is truncated at 3). The electronic energies and the length of the internal coordinate $(q)$ along the reaction path were fitted to a sixthorder polynomial with respect to the new uniform reaction-coordinate scale (because structures calculated along individual reaction path did not occur at the same values of the new reaction-coordinate scale). These functions were Boltzmann-averaged as described previously ${ }^{48}$ and the results were fitted to a sixthorder polynomial to eliminate scatter, which arises because individual structures along reaction path had finite separation. This data is plotted in Fig. 1.

DFT calculations of externally constrained ensembles. All calculations were performed with Gaussian09.B01 using Berny's algorithm to locate stationary points. To ensure reliable compliances, very tight convergence criteria and ultrafine integration grids were used. Thermodynamic corrections to electronic energies of individual conformers were calculated statistical-mechanically in the harmonic oscillator/rigid rotor/ideal gas approximations. ${ }^{57}$ Vibration frequencies $<50 \mathrm{~cm}^{-1}$ were replaced with $50 \mathrm{~cm}^{-1}$ to avoid the artifactually large contribution of such modes to entropy. ${ }^{57}$ Ensembles free energies were calculated as $G_{\min }-\mathrm{RT} \ln \sum g_{\mathrm{i}} e^{-\Delta G_{\mathrm{i}} / R T}$, where $G_{\min }$ is the free energy of the conformational minimum, $\Delta G_{\mathrm{i}}$ is the free energy of conformer $i$ relative to this minimum, and $g_{\mathrm{i}}$ is its degeneracy. Energy barriers separating individual strain-free conformers were $<4 \mathrm{kcal} \mathrm{mol}^{-1}$ (versus $>20 \mathrm{kcal} \mathrm{mol}^{-1}$ for $\Delta G^{\ddagger 0}$ ), justifying calculations of ensemble properties and activation energies by Boltzmann statistics. The basis set superposition error $(1.2 \mathrm{kcal} / \mathrm{mol})$ was estimated as the difference of singleconformer electronic activation energy of the first step of the reduction of $\mathrm{Et}_{2} \mathrm{~S}_{2}$ by $\mathrm{PPh}_{2} \mathrm{CH}_{3}$ calculated with 6-311+G(d) and B3LYP/6-311+ + G(3df, 2pd) basis sets in SMD $^{53}$ (Supplementary Table S15).

We calculated the activation free energy of the first step of $\mathrm{PPh}_{3}$ and $\mathrm{PPh}_{2} \mathrm{CH}_{3}$ reduction of $\mathrm{Et}_{2} \mathrm{~S}_{2}$ coupled to massless infinitely compliant constraining potential (Fig. 7b) by equation (5), ${ }^{3}$ which is derived from the statistical-mechanical formulation of the transition state theory, ${ }^{57}$ where $E_{\mathrm{i}}, T C_{\mathrm{i}}$ and $q_{\mathrm{i}}$ are the electronic energy, thermodynamic correction and the length of the constrained coordinate in conformer $i$ and summation is overall conformational minima that exist in mechanical equilibrium with constraining force $f\left(\ddagger\right.$ signifies the transition state) ${ }^{23}$ This formalism is applicable for constrained molecules in this study, because a molecule plus its constraint are stationary points and an infinitely compliant potential does not perturb the vibration frequencies of the coupled molecule. ${ }^{3}$

$$
\Delta G^{\ddagger}(f)=-\mathrm{R} \operatorname{Tln} \frac{\sum e^{-\frac{E_{i}^{\ddagger}(f)+T C_{i}^{\ddagger}(f)-f \times q_{i}^{\ddagger}(f)}{k T}}}{\sum e^{-\frac{E_{i}(f)+T C_{i}(f)-f \times q_{i}(f)}{k T}}}
$$

At least two strategies exist to calculate the parameters of equation (5): First, direct computation of $E_{\mathrm{i}}(f), T C_{\mathrm{i}}(f)$ and $q_{\mathrm{i}}(f)$ by solving the system of equations $\{\partial E / \partial Q(1) \approx-f, \partial E / \partial Q(i>1)=0\}$, such that the solution satisfies the definition of the local minimum or the first order saddle point. Here $\mathbf{Q}$ is a vector of $3 \mathrm{~N}-6$ non-redundant internal coordinates of the molecule with $\mathbf{Q}(1)$ being the constrained molecular coordinate (note that the system of equations does not generally have a unique solution, that is, even a single conformer would have several geometries that would satisfy the system of equations). This strategy was used successfully $\mathrm{y}^{20-22,24}$ to calculate electronic energies of activation of cyclobutene ring-opening and analogous reactions in single ground and transition-state conformers of small molecules. This approach is particularly useful for MD simulations, but its current implementations remain too expensive for optimizations of thermodynamic states comprising dozens or hundreds of individual conformers.

Alternatively, there is a two-step procedure based on the highly efficient relaxed potential energy scan (rPES) algorithm implemented in Gaussian. In this approach, the electronic energy and thermodynamic corrections are calculated for several values of the constrained distance, $q$, for example, $E_{\mathrm{i}}(q), T C_{\mathrm{i}}(q)$. In Gaussian, the correlation between the length of the constrained coordinate and its restoring force, $q_{\mathrm{i}}$ versus $f_{\mathrm{i}}$ is obtained automatically, that is, $\mathrm{rPES}$ produces both $E_{\mathrm{i}}(q)$ and $E_{\mathrm{i}}(f)$ 
correlations. Depending on the size of the increment of $q$ used in potential energy scans, the $E_{\mathrm{i}}(f)$ and $T C_{\mathrm{i}}(f)$ correlations may be sampled at different values of $f$ for different conformers, for example, electronic energy and thermodynamic corrections for conformer 1 may be calculated at restoring forces of 10,22, 35, 47, $60, \ldots \mathrm{pN}$; whereas the same parameters for conformer 2 may be calculated at restoring forces of $12,30,50,65, \ldots \mathrm{pN}$. As an infinitely compliant potential can impose the same force on all conformers regardless of the length of their constrained distance, discrete values of $E_{\mathrm{i}}\left(f_{\mathrm{i}}\right), T C_{\mathrm{i}}\left(f_{\mathrm{i}}\right)$ and $q_{\mathrm{i}}\left(f_{\mathrm{i}}\right)$ thus obtained were interpolated using the spline algorithm to yield values of $E_{\mathrm{i}}(f), T C_{\mathrm{i}}(f)$ and $q_{\mathrm{i}}(f)$ at the same force for all conformers. For each conformer, the raw scan data (electronic energies, thermodynamic corrections, compliances, $\mathrm{H}_{3} \mathrm{C} \cdots \mathrm{CH}_{3}(q)$ and two $\mathrm{S} \cdots \mathrm{CH}_{2}\left(q_{1}\right)$ separations) were interpolated to obtain uniform force increment of 1 $\mathrm{pN}$ using the interp1 function of Matlab 7.13.0.564. The interpolated data were fitted to a sixth-order polynomial to smooth it.

Conformers of $\mathrm{Et}_{2} \mathrm{~S}_{2}$ and the corresponding transition states were searched systematically, as previously described. ${ }^{25}$ Analytical frequency calculations yielded 0 (reactants) or 1 imaginary frequency (transition states) for all converged structures. Supplementary Tables S16-S18 list key parameters for the conformers. The calculated activation free energy of the first step of reduction of $\mathrm{Et}_{2} \mathrm{~S}_{2}$ with $\mathrm{PPh}_{2} \mathrm{CH}_{3}$ and $\mathrm{PPh}_{3}$ in $\mathrm{H}_{2} \mathrm{O}$ are 15.7 and $19.2 \mathrm{kcal} / \mathrm{mol}$, respectively, versus 14.9 and $17.1 \mathrm{kcal} / \mathrm{mol}$ measured in $0.1 \mathrm{M} \mathrm{NaOH}$ in $\mathrm{H}_{2} \mathrm{O} /$ dioxane $(3: 1 \mathrm{~mol}){ }^{49-51} \mathrm{We}$ calculated the restoring forces of the local coordinate, $f_{l}$ in each conformer of $\mathrm{Et}_{2} \mathrm{~S}_{2}$ as $f \lambda_{c l} l \lambda_{l}{ }^{3}$ where $\lambda_{l}$ is (force-dependent) compliance of local coordinate $l$, and $\lambda_{c l}$ is the coupling coefficient ${ }^{58}$ between this coordinate and the constrained distance. All compliances were derived from analytical Hessians computed at force $f$. Calculations with B3LYP/6-311 + G(d) and B3LYP/6-311+ + G(3df, 2pd) basis sets resulted in nearly identical force-extension curves and the relationship between the stretching and local forces of the ensemble of $\mathrm{Et}_{2} \mathrm{~S}_{2}$ (Supplementary Fig. S5).

The force-induced barrier lowering results exclusively from the decrease in the potential energy of the constraining potential that accompanies the formation of the transition state (Supplementary Fig. S2); the constraining potential destabilizes the transition state to a larger extent than the reactant, accounting for barrierraising contribution of the molecular strain energy.

Molecular compliances were calculated as $\boldsymbol{B} \boldsymbol{H}^{-1} \boldsymbol{B}^{\mathrm{T}}$, where $\boldsymbol{H}$ is the Cartesian Hessians (obtained from frequency calculations on converged geometries), $\boldsymbol{B}$ is a truncated Wilson matrix (having three rows, for the constrained $\mathrm{H}_{3} \mathrm{C} \cdots \mathrm{CH}_{3}$ and the two local coordinates, $\mathrm{S}^{\cdots} \mathrm{CH}_{2}, q_{l}$ ), and ${ }^{-1}$ signifies inversion by the "backslash' numerical algorithm of Matlab.

DFT calculations of the macrocycles. Conformers of all $Z$ and $E$ macrocycles (reactants) were first searched by a Monte-Carlo algorithm with the MMFF force field in VeraChem. The conformers were then reoptimized at BLYP/6-31 + G(d) with density autofitting in gas phase, and unique structures reoptimized at B3LYP/ $6311+\mathrm{G}(\mathrm{d})$ in $\mathrm{H}_{2} \mathrm{O}$-parameterized SMD, followed by wavefunction stability test and analytical frequency calculations. The transition state conformers were generated similarly but with the scissile $\mathrm{S} \cdots \mathrm{S}$ and forming $\mathrm{S} \cdots \mathrm{P}$ bonds frozen at 2.65 and $2.35 \AA$, respectively. The unique conformers were reoptimized at BLYP/6$31+G(d)$ with density autofitting. For the unique geometries, the constraints were removed, analytical frequencies were calculated to guide Berny's search and the geometries were reoptimized at B3LYP/6-31 $+\mathrm{G}(\mathrm{d})$ in $\mathrm{H}_{2} \mathrm{O}$-parameterized SMD, followed by wavefunction stability test ( $E$ isomers only) and analytical frequency calculations. Finally, conformers within $2.5 \mathrm{kcal} / \mathrm{mol}$ of the global conformational minimum of each state were reoptimized at B3LYP/6-311 $+\mathrm{G}(\mathrm{d})$ in $\mathrm{H}_{2} \mathrm{O}$-parameterized SMD. All transition-state conformers manifested a single imaginary frequency between -60 and $-170 \mathrm{~cm}^{-1}$. Supplementary Tables S13 and S14 list the key parameters of the reactant and transition states, and Supplementary Tables S15-S17 list the key parameters of individual conformers of the macrocycles.

\section{References}

1. Black, A. L., Lenhardt, J. M. \& Craig, S. L. From molecular mechanochemistry to stress-responsive materials. J. Mater. Chem. 21, 1655-1663 (2011).

2. Klukovich, H. M., Kean, Z. S., Iacono, S. T. \& Craig, S. L. Mechanically induced scission and subsequent thermal remending of perfluorocyclobutane polymers. J. Am. Chem. Soc. 133, 17882-17888 (2011).

3. Kucharski, T. J. \& Boulatov, R. The physical chemistry of mechanoresponsive polymers. J. Mater. Chem. 21, 8237-8255 (2011).

4. Chen, Y. et al. Mechanically induced chemiluminescence from polymers incorporating a 1,2-dioxetane unit in the main chain. Nat. Chem. 4, 559-562 (2012).

5. Craig, S. L. A tour of force. Nature 487, 176-177 (2012).

6. Cravotto, G. \& Cintas, P. Harnessing mechanochemical effects with ultrasound-induced reactions. Chem. Sci. 3, 295-307 (2012).

7. Kean, Z. S. \& Craig, S. L. Mechanochemical remodeling of synthetic polymers. Polymer 53, 1035-1048 (2012).

8. Wiggins, K. M., Brantley, J. N. \& Bielawski, C. W. Polymer mechanochemistry: force enabled transformations. ACS Macro Lett. 1, 623-626 (2012).

9. Black Ramirez, A. L. et al. Mechanochemical strengthening of a synthetic polymer in response to typically destructive shear forces. Nat. Chem. 5, 757-761 (2013).
10. Kucharski, T. J., Tian, Y., Akbulatov, S. \& Boulatov, R. Chemical solutions for the closed-cycle storage of solar energy. Energy Environ. Sci. 4, 4449-4472 (2011).

11. Lenhardt, J. M. et al. Trapping a diradical transition state by mechanochemical polymer extension. Science 329, 1057-1060 (2010).

12. Suzuki, Y. \& Dudko, O. K. Single-molecule rupture dynamics on multidimensional landscapes. Phys. Rev. Lett. 104, 048101 (2010).

13. Ribas-Arino, J. \& Marx, D. Covalent mechanochemistry: theoretical concepts and computational tools with applications to molecular nanomechanics. Chem Rev. 112, 5412-5487 (2012).

14. Mauguière, F. A. L., Collins, P., Ezra, G. S. \& Wiggins, S. Bond breaking in a Morse chain under tension: Fragmentation patterns, higher index saddles, and bond healing. J. Chem. Phys. 138, 134118 (2013).

15. Hyeon, C. \& Thirumalai, D. Multiple barriers in forced rupture of protein complexes. J. Chem. Phys. 137, 055103 (2012).

16. Franco, I., Schatz, G. C. \& Ratner, M. A. Single-molecule pulling and the folding of donor-acceptor oligorotaxanes: phenomenology and interpretation. J. Chem. Phys. 131, 124902 (2009).

17. Evans, E. Probing the relation between force lifetime and chemistry in single molecular bonds. Annu. Rev. Biophys. Biomol. Struct. 30, 105-128 (2001)

18. Kreuzer, H. J. \& Payne, S. H. Stretching a macromolecule in an atomic force microscope: statistical mechanical analysis. Phys. Rev. E 63, 021906 (2001).

19. Hummer, G. \& Szabo, A. Free energy surfaces from single-molecule force spectroscopy. Acc. Chem. Res. 38, 504-513 (2005).

20. Ribas-Arino, J., Shiga, M. \& Marx, D. Understanding covalent mechanochemistry. Angew. Chem., Int. Ed. 48, 4190-4193 (2009).

21. Kochhar, G. S., Bailey, A. \& Mosey, N. J. Competition between orbitals and stress in mechanochemistry. Angew. Chem., Int. Ed. 49, 7452-7455 (2010).

22. Dopieralski, P., Ribas-Arino, J. \& Marx, D. Force-transformed free-energy surfaces and trajectory-shooting simulations reveal the mechanostereochemistry of cyclopropane ring-opening reactions. Angew. Chem., Int. Ed. 50, 7105-7108 (2011).

23. Hermes, M. \& Boulatov, R. The entropic and enthalpic contributions to forcedependent dissociation kinetics of the pyrophosphate bond. J. Am. Chem. Soc. 133, 20044-20047 (2011).

24. Bailey, A. \& Mosey, N. J. Prediction of reaction barriers and force-induced instabilities under mechanochemical conditions with an approximate model: a case study of the ring opening of 1,3-cyclohexadiene. J. Chem. Phys. 136, 044102 (2012).

25. Tian, Y. \& Boulatov, R. Quantum-chemical validation of the local assumption of chemomechanics for a unimolecular reaction. ChemPhysChem 13, 2277-2281 (2012).

26. Tian, Y. \& Boulatov, R. Comparison of the predictive performance of the BellEvans, Taylor-expansion and statistical-mechanics models of mechanochemistry. Chem. Commun. 49, 4187-4189 (2013).

27. Patel, A. B. et al. Influence of architecture on the kinetic stability of molecular assemblies. J. Am. Chem. Soc. 126, 1318-1319 (2004).

28. Zou, S., Schoenherr, H. \& Vancso, G. J. Force spectroscopy of quadruple H-bonded dimers by AFM: dynamic bond rupture and molecular timetemperature superposition. J. Am. Chem. Soc. 127, 11230-11231 (2005).

29. Kucharski, T. J. et al. Kinetics of thiol/disulfide exchange correlate weakly with the restoring force in the disulfide moiety. Angew. Chem, Int. Ed. 48, 7040-7043 (2009).

30. Yang, Q.-Z. et al. A molecular force probe. Nat. Nanotechnol. 4, 302-306 (2009).

31. Kucharski, T. J., Yang, Q.-Z., Tian, Y. \& Boulatov, R. Strain-dependent acceleration of a paradigmatic $\mathrm{S}_{\mathrm{N}} 2$ reaction accurately predicted by the force formalism. J. Phys. Chem. Lett. 1, 2820-2825 (2010).

32. Akbulatov, S., Tian, Y. \& Boulatov, R. Force-reactivity property of a single monomer is sufficient to predict the micromechanical behavior of its polymer. J. Am. Chem. Soc. 134, 7620-7623 (2012).

33. Schmidt, S. W., Beyer, M. K. \& Clausen-Schaumann, H. Dynamic strength of the silicon-carbon bond observed over three decades of force-loading rates. J. Am. Chem. Soc. 130, 3664-3668 (2008).

34. Kersey, F. R., Yount, W. C. \& Craig, S. L. Single-molecule force spectroscopy of bimolecular reactions: System homology in the mechanical activation of ligand substitution reactions. J. Am. Chem. Soc. 128, 3886-3887 (2006).

35. Tobe, M. L. \& Burgess, J. Inorganic Reaction Mechanisms (Pearson, 1999).

36. Rickborn, B. in Organic Reactions Vol. 52 (eds Paquette, L. A.) (Wiley, 1998).

37. Merkel, R., Nassoy, P., Leung, A., Ritchie, K. \& Evans, E. Energy landscapes of receptor-ligand bonds explored with dynamic force spectroscopy. Nature 397, 50-53 (1999).

38. Evans, E., Leung, A., Hammer, D. \& Simon, S. Chemically distinct transition states govern rapid dissociation of single l-selectin bonds under force. Proc. Natl Acad. Sci. USA 98, 3784-3789 (2001).

39. Williams, P. M. et al. Hidden complexity in the mechanical properties of titin. Nature 422, 446-449 (2003). 
40. Derenyi, I., Bartolo, D. \& Ajdari, A. Effects of intermediate bound states in dynamic force spectroscopy. Biophys. J. 86, 1263-1269 (2004).

41. Imparatoa, A. \& Peliti, L. Kinetic barriers in RNA unzipping. Eur. Phys. J. B 39, 357-363 (2004).

42. Rico, F. \& Moy, V. T. Energy landscape roughness of the streptavidin-biotin interaction. J. Mol. Recognit. 20, 495-501 (2007).

43. Garcia-Manyes, S., Liang, J., Szoszkiewicz, R., Kuo, T.-L. \& Fernández, J. M. Force-activated reactivity switch in a bimolecular chemical reaction. Nat. Chem. 1, 236-242 (2009).

44. Yew, Z. T., Schlierf, M., Rief, M. \& Paci, E. Direct evidence of the multidimensionality of the free-energy landscapes of proteins revealed by mechanical probes. Phys. Rev. E 81, 031923 (2010).

45. Huang, Z. \& Boulatov, R. Chemomechanics with molecular force probes. Pure Appl. Chem. 82, 931-951 (2010).

46. Boulatov, R. Demonstrated leverage. Nat. Chem. 5, 84-86 (2013).

47. Xia, F., Bronowska, A. K., Cheng, S. \& Grater, F. Base-catalyzed peptide hydrolysis is insensitive to mechanical stress. J. Phys. Chem. B 115, 10126-10132 (2011)

48. Akbulatov, S., Tian, Y., Kapustin, E. \& Boulatov, R. Model studies of the kinetics of ester hydrolysis under stretching force. Angew. Chem., Int. Ed. 52, 6992-6995 (2013)

49. Overman, L. E., Matzinger, D., O’Connor, E. M. \& Overman, J. D. Nucleophilic cleavage of the sulfur-sulfur bond by phosphorus nucleophiles. Kinetic study of the reduction of aryl disulfides with triphenylphosphine and water. J. Am. Chem. Soc. 96, 6081-6089 (1974).

50. Overman, L. E. \& Petty, S. T. Nucleophilic cleavage of the sulfur-sulfur bond by phosphorus nucleophiles. III. Kinetic study of the reduction of a series of ethyl aryl disulfides with triphenylphosphine and water. J. Org. Chem. 40, 2779-2782 (1975).

51. Overman, L. E. \& O'Connor, E. M. Nucleophilic cleavage of the sulfur-sulfur bond by phosphorus nucleophiles. IV. Kinetic study of the reduction of alkyl disulfides with triphenylphosphine and water. J. Am. Chem. Soc. 98, 771-775 (1976).

52. Dmitrenko, O., Thorpe, C. \& Bach, R. D. Mechanism of $\mathrm{S}_{\mathrm{N}} 2$ disulfide bond cleavage by phosphorus nucleophiles. Implications for biochemical disulfide reducing agents. J. Org. Chem. 72, 8298-8307 (2007).
53. Marenich, A. V., Cramer, C. J. \& Truhlar, D. G. Universal solvation model based on solute electron density and on a continuum model of the solvent defined by the bulk dielectric constant and atomic surface tensions. J. Phys. Chem. B 113, 6378-6396 (2009).

54. Huang, Z. \& Boulatov, R. Chemomechanics: Chemical kinetics for multiscale phenomena. Chem. Soc. Rev. 40, 2359-2384 (2011).

55. Dopieralski, P. et al. The Janus-faced role of external forces in mechanochemical disulfide bond cleavage. Nat. Chem. 5, 685-691 (2013).

56. Gans, P. Data Fitting in the Chemical Sciences by the Method of Least Squares (John Wiley \& Sons, 1992).

57. Cramer, C. J. Essentials of Computational Chemistry 2nd edn (Wiley, 2004).

58. Baker, J. A critical assessment of the use of compliance constants as bond strength descriptors for weak interatomic interactions. J. Chem. Phys. 125, 014103 (2006).

\section{Acknowledgements}

The work was supported by the University of Liverpool and the US NSF (CHE-0748281 and TG-CHE090066).

\section{Author contributions}

Y.T. performed all quantum-chemical computations. T.J.K. performed all kinetic measurements and contributed to the synthesis of the disulphides. Q-Z.Y. developed the synthetic methods. R.B. conceived of and supervised the study and wrote the manuscript.

\section{Additional information}

Supplementary Information accompanies this paper at http://www.nature.com/ naturecommunications

Competing financial interests: The authors declare no competing financial interests.

Reprints and permission information is available online at http://npg.nature.com/ reprintsandpermissions/

How to cite this article: Tian, Y. et al. Model studies of force-dependent kinetics of multi-barrier reactions. Nat. Commun. 4:2538 doi: 10.1038/ncomms3538 (2013). 